\title{
Mechanical thrombectomy in basilar artery occlusion: influence of reperfusion on clinical outcome and impact of the first-line strategy (ADAPT vs stent retriever)
}

\author{
Benjamin Gory, MD, PhD, ${ }^{1}$ Mikael Mazighi, MD, PhD, ${ }^{2-5}$ Raphael Blanc, MD, MSc, ${ }^{2}$ \\ Julien Labreuche, BST, ${ }^{6}$ Michel Piotin, MD, PhD, ${ }^{2}$ Francis Turjman, MD, PhD, ${ }^{7}$ and \\ Bertrand Lapergue, MD, PhD, ${ }^{8}$ on behalf of the Endovascular Treatment in Ischemic Stroke \\ (ETIS) Research Investigators
}

\begin{abstract}
1Department of Diagnostic and Interventional Neuroradiology, INSERM U947, University Hospital of Nancy, University of Lorraine, Nancy; ${ }^{2}$ Department of Interventional Neuroradiology, Rothschild Foundation, Paris; ${ }^{3} \mathrm{DHU}$ NeuroVasc Sorbonne Paris Cité; ${ }^{4}$ Paris Diderot University (Paris 7), Paris; ${ }^{5}$ Laboratory of Vascular Translational Science, INSERM U1148, Paris; ${ }^{6}$ Department of Biostatistics, EA2694-Santé publique: épidémiologie et qualité des soins, Lille University, Lille; 'Department of Interventional Neuroradiology, Hospices Civils de Lyon; and ${ }^{8}$ Department of Neurology, Stroke Center, Foch Hospital, Université de Versailles Saint-Quentin-en-Yvelines, Suresnes, France
\end{abstract}

OBJECTIVE Several randomized trials have been focused on patients with anterior circulation stroke, whereas few data on posterior circulation stroke are available. Thus, new mechanical thrombectomy (MT) strategies, including a directaspiration first-pass technique (ADAPT), remain to be evaluated in basilar artery occlusion (BAO) patients. The authors here assessed the influence of reperfusion on outcome in BAO patients and examined whether ADAPT improves the reperfusion rate compared with stent retriever devices.

METHODS Three comprehensive stroke centers prospectively collected individual data from BAO patients treated with MT. Baseline characteristics as well as radiographic and clinical outcomes were compared between the 2 MT strategies. The primary outcome measure was the rate of successful reperfusion, defined as a modified Thrombolysis in Cerebral Infarction (mTICl) grade of 2b-3. Favorable outcome was defined as a 90-day modified Rankin Scale score of 0-2.

RESULTS Among the 100 adult patients included in the study, 46 were treated with first-line ADAPT (median age 61 years, IQR 53-71 years; stent-retriever rescue therapy was secondarily used in 12 [26.1\%]) and 54 were treated with a primary stent retriever (median age 67 years, IQR 53-78 years). There was no difference in baseline characteristics between the 2 treatment groups, except for the rate of diabetes ( $19.6 \%$ vs $5.7 \%$, respectively, $p=0.035$ ). Successful reperfusion was achieved in $79 \%$ of the overall study sample. Overall, the rate of favorable outcome was $36.8 \%$ and 90-day all-cause mortality was $44.2 \%$. Successful reperfusion positively impacted favorable outcome (OR $4.57,95 \%$ $\mathrm{Cl} 1.24-16.87, p=0.023$ ). A nonsignificant trend toward a higher successful reperfusion rate (unadjusted OR $2.56,95 \%$ $\mathrm{Cl} 0.90-7.29, p=0.071$ ) and a significantly higher rate of complete reperfusion ( $\mathrm{mTICl}$ grade 3 ; unadjusted OR 2.59, $95 \% \mathrm{Cl} 1.14-5.86, p=0.021$ ) was found in the ADAPT group. The procedure duration was also significantly lower in the ADAPT group (median 45 minutes, IQR 34 to 62 minutes vs 56 minutes, IQR 40 to 90 minutes; $p=0.05$ ), as was the rate of periprocedural complications $(4.3 \%$ vs $25.9 \%, p=0.003)$. Symptomatic intracranial hemorrhage $(0.0 \%$ vs $4.0 \%, p=$ $0.51)$ and 90 -day all-cause mortality $(46.7 \%$ vs $42.0 \%, p=0.65)$ were similar in the 2 groups.

CONCLUSIONS Among BAO patients, successful reperfusion is a strong predictor of a 90 -day favorable outcome, and the choice of ADAPT as the first-line strategy achieves a significantly higher rate of complete reperfusion with a shorter procedure duration.

https://thejns.org/doi/abs/10.3171/2017.7.JNS171043

KEY WORDS acute stroke; ADAPT; stent retriever; thrombectomy; basilar artery occlusion; aspiration; reperfusion; vascular disorders

ABBREVIATIONS ADAPT $=$ a direct-aspiration first-pass technique; ASPECTS = Alberta Stroke Programme Early CT Score; BAO = basilar artery occlusion; $m$ RS = modified Rankin Scale; MT = mechanical thrombectomy; $\mathrm{mTICl}=$ modified Thrombolysis in Cerebral Ischemia; NIHSS = National Institutes of Health Stroke Scale.

SUBMITTED April 26, 2017. ACCEPTED July 6, 2017.

INCLUDE WHEN CITING Published online January 12, 2018; DOI: 10.3171/2017.7.JNS171043. 
$\mathrm{M}$ ECHANICAL thrombectomy (MT) has been validated through 6 international randomized controlled trials in patients with acute ischemic stroke caused by occlusion of the arteries of the proximal anterior circulation. ${ }^{2,12}$ However, patients with basilar artery occlusion (BAO) were excluded from 5 of these trials, and only a few such patients were included in the THRACE (THRobectomie des Artères CErebrales) trial. ${ }^{2}$ In patients with $\mathrm{BAO}$, prognosis remains severe with a high mortality rate, especially in the absence of early reperfusion..$^{27}$ Pending the results of randomized trials,,${ }^{17,31}$ there is, to date, no consensus regarding the management of these patients, especially in view of results from the BASICS (Basilar Artery International Cooperation Study) registry reporting no benefit from the intraarterial approach compared with medical treatment. ${ }^{25}$ It is noteworthy that no modern MT devices improving reperfusion and clinical outcomes $^{22,24}$ were used in the BASICS.

Recent data on BAO patients treated with stent retrievers reveal that clinical outcomes with a wide range of functional independence can be achieved. ${ }^{11}$ The latest treatment, a direct-aspiration first-pass technique (ADAPT), involves first-line aspiration to remove the thrombus through a large-bore aspiration catheter. ${ }^{30}$ These large catheters are important tools for MT of a large-vessel occlusion allowing high rates of reperfusion mainly reported in the anterior circulation (Gory B, et al: A direct aspiration first pass technique [ADAPT] for acute ischemic stroke therapy: a systematic review and meta-analysis; submitted, 2017). ${ }^{16}$

We, therefore, assessed the influence of reperfusion on outcome in BAO patients and, by pooling prospective individual data from 3 comprehensive stroke centers, examined whether ADAPT improves the reperfusion rate compared with stent retriever devices.

\section{Methods \\ Study Design and Population}

In this study, we conducted a retrospective analysis of our prospectively collected, large-vessel occlusion stroke, endovascular database to identify patients presenting with BAO between March 2010 and October 2016 at 3 comprehensive stroke centers (Endovascular Treatment in Ischemic Stroke [ETIS] registry). Detailed materials and methods have been reported elsewhere. ${ }^{16}$ The local ethics committee approved this research protocol.

There was no prespecified protocol for the treatment of BAO strokes at our centers during the study period. The decision to treat or not to treat any given patient with $\mathrm{BAO}$ on presentation was left to the attending neuroendovascular specialist's discretion in agreement with the stroke team caring for the patient and the patient's family.

\section{Endovascular Procedure}

The aim of the MT procedure was to achieve successful reperfusion (modified Thrombolysis In Cerebral Ischemia $[\mathrm{mTICI}]$ grade $\geq 2 \mathrm{~b}$ ) of the territory of the occluded vessel as quickly as possible. ${ }^{33}$ The first-line device was chosen at the neurointerventionist's discretion, either a stent retriever or a large-bore aspiration catheter (ADAPT; Fig. 1), with the possibility of switching to another strat- egy in the case of reperfusion failure (mTICI grade $<2 b$ ) with the first approach.

\section{Eligibility and Data Collection}

Inclusion criteria were as follows: 1) acute stroke symptoms with no upper age or National Institutes of Health Stroke Scale (NIHSS) score limit; 2) BAO confirmed on angiography with time from the "last known normal" patient status to treatment of less than 24 hours; 3) reperfusion using stent retrievers or primary aspiration with largebore catheters (ADAPT); and 4) thrombectomy performed either as the primary treatment or as an add-on to intravenous thrombolysis with recombinant tissue plasminogen activator. Intravenous thrombolysis was administered in eligible patients in accordance with current management guidelines. No specific exclusion criteria were applied. The institutional review board at each center approved the study.

The following variables were collected: age; sex; medical history including main vascular risk factors (hypertension, diabetes mellitus, hypercholesterolemia, and current smoking); initial stroke severity as expressed by the admission NIHSS score; initial imaging modality at admission and the Alberta Stroke Programme Early CT Score (ASPECTS); prior use of intravenous thrombolysis; and type of MT devices. Procedure was monitored according to procedure-related complications, degree of reperfusion assessed per the mTICI scale, ${ }^{26}$ time from symptom onset to successful reperfusion, and conventional angiography at the end of thrombectomy. The mTICI grade was scored as follows: 0 (no recanalization), no perfusion or anterograde flow beyond the occlusion site; 1 (minimal recanalization), contrast medium passes the area of occlusion but fails to opacify the entire cerebral bed distal to the obstruction during the angiographic run; 2 (partial recanalization), 2a: partial filling, $<50 \%$ of territory visualized, $2 \mathrm{~b}$ : partial filling, $\geq 50 \%$ of territory visualized; and 3 (complete recanalization), total reperfusion with normal filling. Clinical outcome was assessed using the modified Rankin Scale (mRS) score, as were intracranial hemorrhage at 24 hours on cerebral imaging (MRI or CT) and mortality. The mRS score was assigned as follows: 0 , no symptoms; 1 , no clinically relevant disability; 2 , slight disability (able to look after own affairs without assistance but not to a full extent); 3 , moderate disability (requires some help but able to walk unassisted); 4 , moderately severe disability (unable to attend to own bodily needs or to walk without assistance); 5, severe disability (requires constant nursing care); and 6, dead. Intracranial hemorrhage was classified as hemorrhagic infarction or parenchymal hemorrhage. ${ }^{13}$ Stroke etiology was defined according to the Trial of Org 10172 in Acute Stroke Treatment (TOAST) classification. ${ }^{1}$ Functional independence and mortality were assessed at 90 days during face-to-face interviews or via telephone calls by trained research nurses unaware of the study group assignments?

\section{Outcome Measures}

The primary efficacy outcome measure was successful reperfusion, defined as mTICI grade $2 b-3$ at the end 

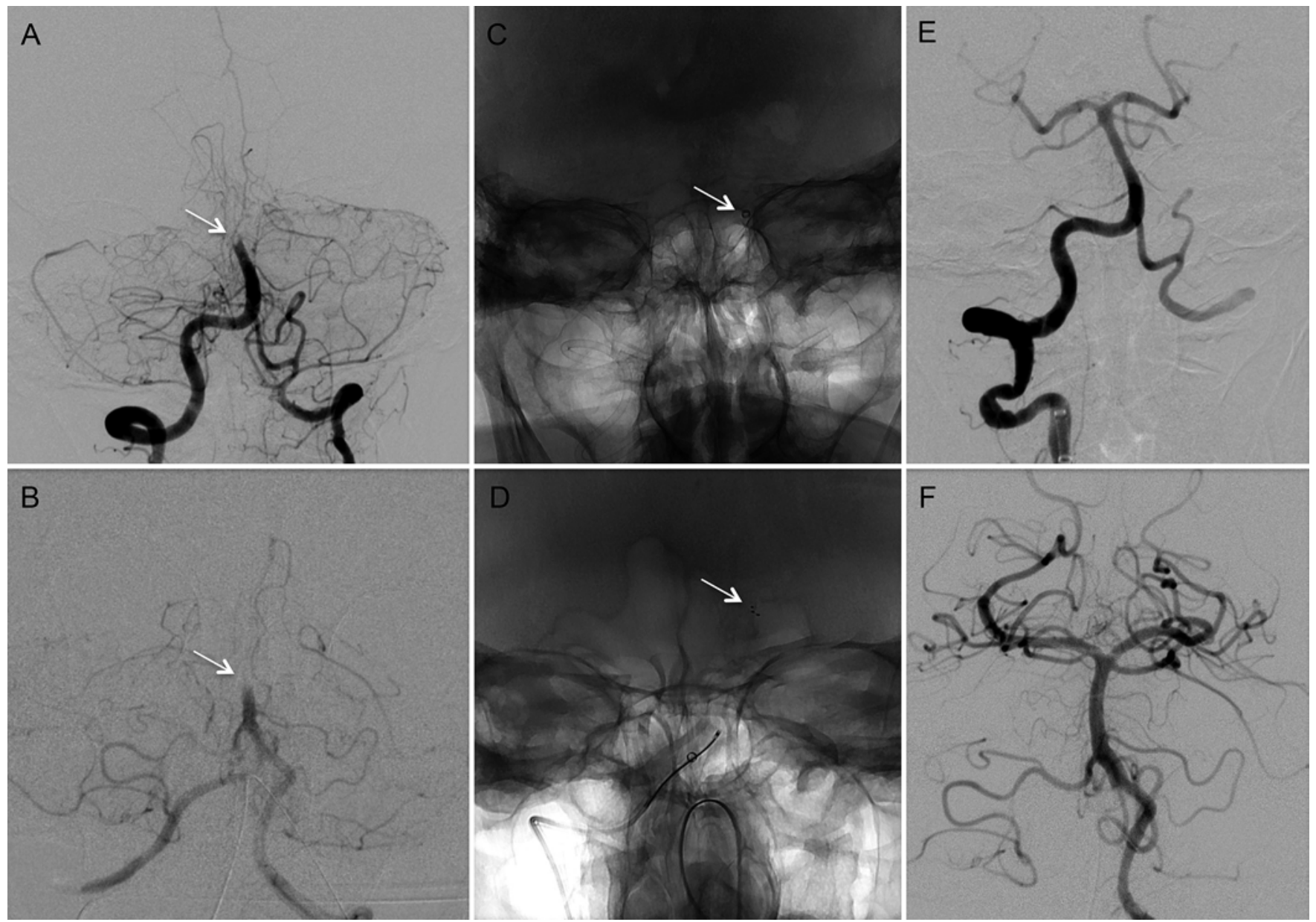

FIG. 1. Demonstration of ADAPT and a stent retriever thrombectomy in BAO. Pretreatment anterior angiograms $(\mathbf{A}$ and $\mathbf{B})$ showing a complete BAO (arrows). Anterior native images showing the large-bore aspiration catheter (arrow, C) and stent retriever device (arrow, D) in the BA during the procedure. Postthrombectomy anterior angiograms (E and F) showing complete recanalization of the BA.

of the procedure, as assessed by the treating physician. Secondary efficacy outcomes included complete reperfusion (mTICI grade 3), change in NIHSS score at 24 hours, favorable outcome (defined as 90-day mRS score 0-2), and all-cause mortality at 90-days. Safety outcomes of interest included procedural complications, intracranial hemorrhage complication on brain imaging at 24 hours, and symptomatic intracranial hemorrhage (defined as any hemorrhage on follow-up imaging associated with an increase of $\geq 4$ points in the NIHSS score according to the European Co-operative Acute Stroke Study-II [ECASSII] criteria). ${ }^{13}$

\section{Statistical Analysis}

Quantitative variables are expressed as medians (interquartile range), and categorical variables are expressed as numbers (percentage). Normality of distributions was assessed using histograms and the Shapiro-Wilk test.

Bivariate comparisons between the 2 study groups (ADAPT vs stent retriever) were made using the chi-square test (or Fisher's exact test when the expected cell frequency was $<5$ ) for categorical variables, the Student t-test for
Gaussian continuous variables, and the Mann-Whitney Utest for non-Gaussian continuous and ordinal categorical variables, as appropriate. Differences (ADAPT vs stent retriever group) in binary efficacy outcomes (successful reperfusion, complete reperfusion, favorable outcome, and 90-day mortality) between the 2 groups were expressed as odds ratios, and the difference in the 24-hour NIHSS change was expressed as a standardized difference calculated on rank-transformed data using nonparametric ANCOVA including the admission NIHSS score as a covariable; ${ }^{5}$ the $95 \%$ confidence interval of the standardized difference was calculated using the bootstrap method (2000 bootstrap samples). ${ }^{8}$ Comparisons between efficacy outcomes were further adjusted for prespecified confounding factors (age and admission NIHSS) and baseline differences (at $\mathrm{p}<0.10$ in bivariate analyses) by using logistic regression models for binary outcomes and nonparametric ANCOVA for the change in the NIHSS score. Because of missing data on outcomes (favorable outcome and mortality, 5\%; change in NIHSS, $21 \%$ ) and confounding factors (5\%), we performed sensitivity analyses using a multiple imputation approach to handle missing values. 


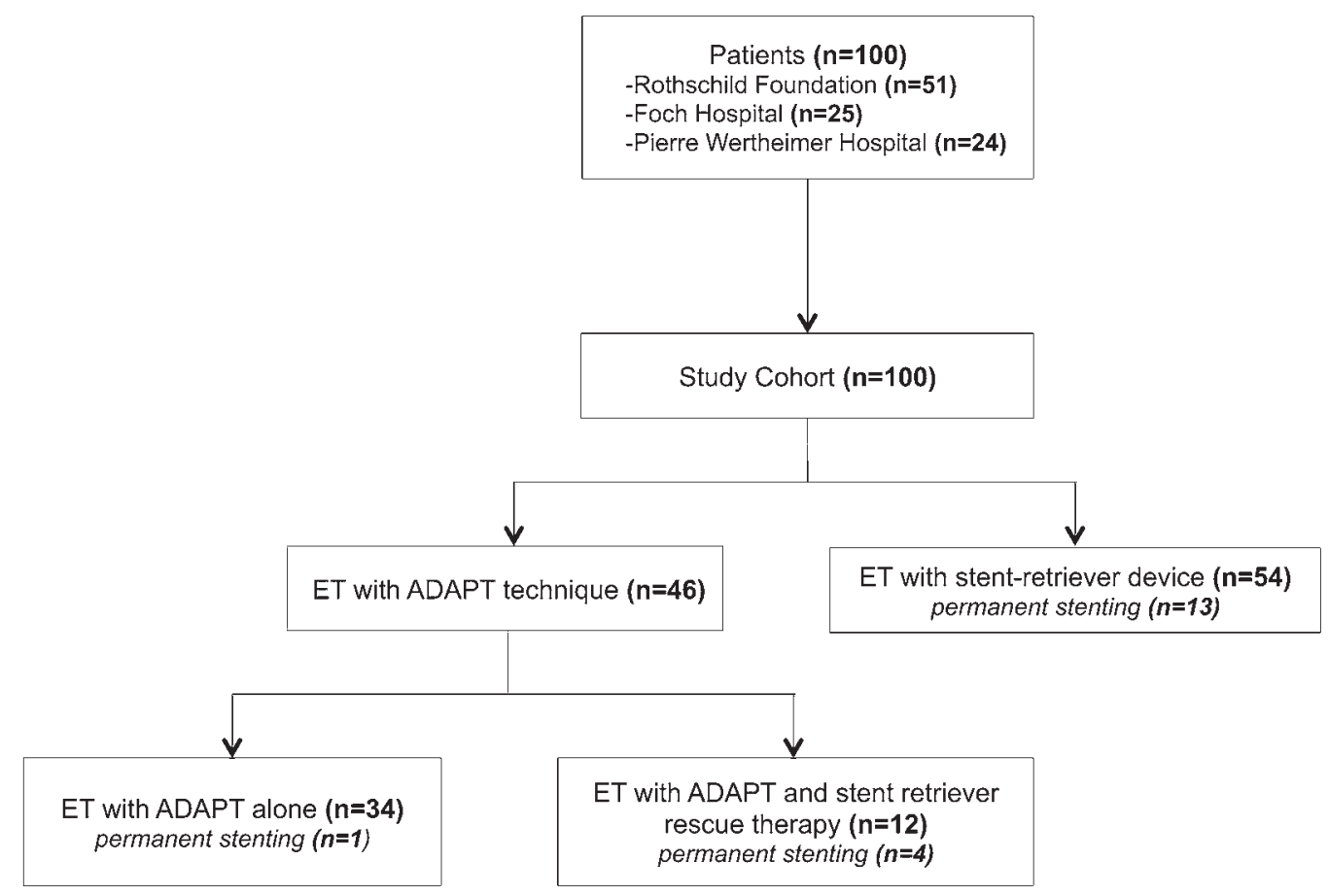

FIG. 2. Flowchart of patient selection. ET = endovascular treatment.

Missing data were imputed under the missing-at-random assumption by using the regression-switching approach (chained equation with $\mathrm{m}=10$ imputations obtained using $\mathrm{R}$ statistical software version 3.03). Imputation procedure was performed under the missing-at-random assumption using all baseline characteristics and the study outcomes with a predictive mean matching method for continuous variables and multinomial or binary logistic regression model for categorical variables. ${ }^{3}$ Estimates obtained in the different imputed data sets were combined using Rubin's rules. ${ }^{23}$

Finally, among the overall patient population, we studied the impact of successful reperfusion on a favorable outcome by using logistic regression analysis before and after adjustment for first-line treatment approach, age, and admission NIHSS score. Statistical testing was done at the 2 -tailed $\alpha$ level of 0.05 . Data were analyzed using SAS software version 9.3 (SAS Institute).

\section{Results}

\section{Baseline Characteristics}

During the study period, 100 adults with BAO were treated with MT at the 3 participating centers, with a median delay of 315 minutes (IQR 225 to 382 minutes; Fig. 2) from symptom onset to groin puncture. Patients' baseline characteristics are described for the overall study sample and according to the first-line endovascular approach in Table 1. A total of 46 patients were treated with primary aspiration, and the remaining 54 patients were treated with stent retrievers. Overall, the median age was 65 years (IQR 53 to 77 years), $61 \%$ of the patients were men, and the median pretreatment NIHSS score was 16 (IQR 11 to 29). Forty-five patients received intravenous thrombolysis prior to endovascular treatment within a median delay of 170 minutes from symptom onset. Except for a lower rate of diabetes in the patients treated with a stent retriever $(5.7 \%$ vs $19.6 \%, p=0.035)$, there were no significant differences in baseline characteristics between the 2 treatment groups. Although the difference did not reach the significance level, the delay from symptom onset to groin puncture was longer in the ADAPT group than in the stent retriever group.

In the ADAPT group, the overall device cost was $\$ 229,479$ ( $\$ 175,484$ for primary aspiration and $\$ 53,995$ dollars for stent-retriever rescue therapy). In the stent retriever group, the overall device cost was $\$ 249,727$ dollars $(\$ 242,978$ for primary stent retriever thrombectomy and $\$ 6749$ for rescue devices).

\section{Procedural Characteristics and Complications}

Table 2 shows the procedural characteristics overall and according to the first-line endovascular approach. Most of the patients were treated under general anesthesia (84.0\%), with no difference according to the first-line approach utilized. Primary aspiration was performed mostly with a 5MAX ACE catheter (65.2\%), and primary stent retriever thrombectomy was performed mostly with the Solitaire device $(77.8 \%)$. Rescue treatment after the firstline strategy was more often necessary in the ADAPT group than in the stent retriever group $(26.1 \%$ vs $3.7 \%$, p $=0.001$.

Procedural complications occurred in $16 \%$ of patients (95\% CI 9.4\%-24.7\%) and were significantly more frequent in the stent retriever group than in the ADAPT group $(25.9 \%$ vs $4.3 \%, \mathrm{p}=0.003)$. Embolization to a new territory was the most frequent procedural complication, 
TABLE 1. Baseline characteristics of 100 patients treated for BAO

\begin{tabular}{|c|c|c|c|c|}
\hline \multirow[b]{2}{*}{ Characteristic } & \multirow[b]{2}{*}{ Overall } & \multicolumn{2}{|c|}{ First-Line Endovascular Approach } & \multirow{2}{*}{$\begin{array}{c}p \\
\text { value }\end{array}$} \\
\hline & & ADAPT & Stent Retriever & \\
\hline No. of patients & 100 & 46 & 54 & \\
\hline Median age in yrs & $65(53-77)$ & $61(53-71)$ & $67(53-78)$ & 0.51 \\
\hline Men & $61 / 100(61.0)$ & $27 / 46(58.7)$ & $34 / 54(63.0)$ & 0.66 \\
\hline Hypertension & $47 / 99(47.5)$ & $25 / 46(54.3)$ & $22 / 53(41.5)$ & 0.20 \\
\hline Hypercholesterolemia & $27 / 98(27.6)$ & $14 / 45(31.1)$ & $13 / 53(24.5)$ & 0.47 \\
\hline Diabetes & $12 / 99(12.1)$ & $9 / 46(19.6)$ & $3 / 53(5.7)$ & 0.035 \\
\hline Current smoker & $25 / 89(28.1)$ & $13 / 43(30.2)$ & $12 / 46(26.1)$ & 0.66 \\
\hline Antithrombotic medications & $35 / 100(35.0)$ & $19 / 46(41.3)$ & $16 / 54(29.6)$ & 0.22 \\
\hline Median admission NIHSS score* & $16(11-29)$ & $14(9-25)$ & $20(11-30)$ & 0.29 \\
\hline MRI & $73 / 100(73.0)$ & $36 / 46(78.3)$ & $37 / 54(68.5)$ & 0.27 \\
\hline Median ASPECTS & $7(6-8)$ & $7(6-9)$ & $8(6-8)$ & 0.60 \\
\hline Patients w/ ASPECTS $<7$ & 28/91 (30.8) & $14 / 44(31.8)$ & $14 / 47(29.8)$ & 0.83 \\
\hline Prior use of IV thrombolysis & $45 / 100(45.0)$ & $23 / 46(50.0)$ & $22 / 54(40.7)$ & 0.35 \\
\hline Median delay from symptom onset to IV thrombolysis in mins† & $170(127-240)$ & $170(130-250)$ & $173(125-230)$ & 0.94 \\
\hline \multicolumn{5}{|l|}{ Etiology } \\
\hline Large-artery atherosclerosis & $30 / 91(33.0)$ & $14 / 43(32.6)$ & $16 / 48(33.3)$ & 0.52 \\
\hline Cardioembolic & 29/91 (31.9) & $16 / 43(37.2)$ & $13 / 48(27.1)$ & \\
\hline Other & $32 / 91(35.2)$ & $13 / 43(30.2)$ & $19 / 48(39.6)$ & \\
\hline Median delay from symptom onset to groin puncture in mins $\ddagger$ & $315(225-382)$ & $342(241-440)$ & $276(198-355)$ & 0.081 \\
\hline \multicolumn{5}{|c|}{$\begin{array}{l}\text { IV }=\text { intravenous. } \\
\text { Values are expressed as the number/total number (\%) or median (interquartile range). } \\
\text { * The NIHSS score is defined as } 42 \text { in the setting of coma, a locked-in state, or tetraparesis (missing values for } 2 \text { patients). } \\
\dagger \text { Calculated among patients treated with intravenous thrombolysis prior to endovascular treatment (missing values for } 1 \text { patient). } \\
\text { † Missing values for } 3 \text { patients. }\end{array}$} \\
\hline
\end{tabular}

occurring in $11 \%$ of cases overall. Hemorrhagic complications at 24 hours occurred in $15.7 \%$ of patients $(95 \%$ CI $8.8 \%-25.0 \%$ ) with no significant difference between firstline endovascular approaches $(12.8 \%$ vs $18.0 \%, \mathrm{p}=0.51)$.

\section{Angiographic and Clinical Efficacy Outcomes}

Figure 3 shows the distribution of reperfusion grades at the end of procedure and 90-day clinical outcome, according to treatment group. Overall, successful reperfusion was achieved in $79.0 \%$ of the patients $(95 \%$ CI $69.7 \%-$ $86.5 \%$ ). Secondary outcome rates were $42.0 \%$ (95\% CI $32.2 \%-52.3 \%$ ) for complete reperfusion, $36.8 \%$ (95\% CI $27.1 \%-47.4 \%$ ) for a favorable outcome, and $44.2 \%$ for 90 day all-cause mortality (95\% CI $34.0 \%-54.8 \%$ ). The median 24-hour decrease in the NIHSS score was 3 points (IQR -10 to 4 points; Table 3 ).

Compared with the stent retriever group, the ADAPT group showed a nonsignificant trend toward a higher successful reperfusion rate, with an unadjusted OR of 2.56 (95\% CI 0.90-7.29, p = 0.071; Tables 3 and 4). The median time from groin puncture to successful reperfusion was lower in the ADAPT group than in the stent retriever group (45 minutes [IQR 34 to 62 minutes] vs 56 minutes [IQR 40 to 90 minutes], $\mathrm{p}=0.050$ ). Regarding other secondary efficacy outcomes, only a significantly higher rate of complete reperfusion was found in favor of the first-line aspiration approach (unadjusted OR 2.59, 95\% CI 1.14-5.86, p $=0.021)$. Similar results were found after adjustment for center, age, admission NIHSS score, diabetes, and time from symptom onset to groin puncture, using multiple imputations to handle missing outcomes and covariates.

\section{Successful Reperfusion and Favorable Outcome}

In the 2 groups pooled, patients with successful reperfusion had a favorable outcome (32 patients [43.2\%]) more often than the patients without successful reperfusion (14.3\%; OR 4.57, 95\% CI 1.24-16.87, $\mathrm{p}=0.023$ ). The impact of successful reperfusion on favorable outcome was unchanged after adjustment for the first-line endovascular approach, age, and admission NIHSS score (adjusted OR $5.64,95 \%$ CI $1.32-24.06)$.

\section{Discussion}

This analysis of pooled individual data shows that in $\mathrm{BAO}$ patients, successful reperfusion is a strong predictor of a favorable 90-day outcome and that the choice of ADAPT as the first-line endovascular strategy improves the rate of complete reperfusion and reduces procedure duration compared with the stent retriever technique. In addition, embolization to a new territory occurred more frequently in patients treated with a stent retriever.

This study is one of the largest multicenter series dealing with outcomes of modern MT in patients with angiographically proven BAO. Our analysis confirms that high reperfusion rates can be achieved with the use of modern 
TABLE 2. Procedural characteristics and complications in 100 patients treated for BAO

\begin{tabular}{|c|c|c|c|c|}
\hline \multirow[b]{2}{*}{ Parameter } & \multirow[b]{2}{*}{ Overall } & \multicolumn{2}{|c|}{ First-Line Endovascular Approach } & \multirow[b]{2}{*}{$\mathrm{p}$ Value } \\
\hline & & ADAPT & Stent Retriever & \\
\hline No. of patients & 100 & 46 & 54 & \\
\hline General anesthesia & $84 / 100(84.0)$ & $38 / 46(82.6)$ & $46 / 54(85.2)$ & 0.73 \\
\hline \multicolumn{5}{|l|}{ First-line endovascular device } \\
\hline \multicolumn{5}{|l|}{ Aspiration device } \\
\hline 5MAX ACE & - & $30 / 46(65.2)$ & - & - \\
\hline $5 \mathrm{MAX}$ & - & $8 / 46(17.4)$ & - & - \\
\hline Other aspiration device* & - & $8 / 46(17.4)$ & - & - \\
\hline \multicolumn{5}{|l|}{ Stent retriever device } \\
\hline Solitaire & - & - & $42 / 54(77.8)$ & - \\
\hline Trevo & - & - & $7 / 54(13.0)$ & - \\
\hline Other stent retriever device $†$ & - & - & $5 / 54(9.3)$ & - \\
\hline Rescue therapy $\ddagger$ & $14 / 100(14.0)$ & $12 / 46(26.1)$ & $2 / 54(3.7)$ & 0.001 \\
\hline No. of total passes§ & $2(1-3)$ & $2(1-3)$ & $2(1-3)$ & 0.43 \\
\hline 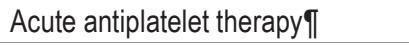 & 13/99 (13.1) & $5 / 46(10.9)$ & $8 / 53(15.1)$ & 0.53 \\
\hline Permanent stenting** & $18 / 100(18.0)$ & $5 / 46(10.9)$ & $13 / 54(24.1)$ & 0.087 \\
\hline Intracranial angioplasty & $21 / 100(21.0)$ & $11 / 46(23.9)$ & 10/54 (18.5) & 0.51 \\
\hline Any procedural complication & $16 / 100(16.0)$ & $2 / 46(4.3)$ & $14 / 54(25.9)$ & 0.003 \\
\hline New-territory embolic event & $11 / 100(11.0)$ & $1 / 46(2.2)$ & 10/54 (18.5) & \\
\hline Vessel perforation & $4 / 100(4.0)$ & $1 / 46(2.2)$ & $3 / 54(5.6)$ & \\
\hline Arterial dissection & $1 / 100(1.0)$ & $0 / 46(0.0)$ & $1 / 54(1.9)$ & \\
\hline Any hemorrhagic complication & $14 / 89(15.7)$ & $5 / 39(12.8)$ & $9 / 50(18.0)$ & 0.51 \\
\hline $\mathrm{slCH}$ & $2 / 89(2.2)$ & $0 / 39(0.0)$ & $2 / 50(4.0)$ & - \\
\hline $\mathrm{SAH}$ & $1 / 89(1.1)$ & $0 / 39(0.0)$ & $1 / 50(2.0)$ & - \\
\hline $\mathrm{PH} 1$ & $3 / 89(3.4)$ & $2 / 39(5.1)$ & $1 / 50(2.0)$ & - \\
\hline $\mathrm{PH} 2$ & $1 / 89(1.1)$ & $0 / 39(0.0)$ & $1 / 50(2.0)$ & - \\
\hline
\end{tabular}

$\mathrm{PH} 1=$ parenchymal hematoma type 1; $\mathrm{PH} 2$ = parenchymal hematoma type 2; $\mathrm{SAH}=$ subarachnoid hemorrhage; sICH = symptomatic intracranial hemorrhage.

Values are expressed as the number/total number (\%) or median (interquartile range).

* Catheter 3MAX (1 patient), catheter ACE64 (2 patients), Catheter ARC (3 patients), Catheter Navien5F (1 patient), and not indicated (1 patient).

$\dagger$ Stent retriever Capture (3 patients), Revive (2 patients).

$\ddagger$ Rescue therapy was defined as the use of another endovascular strategy after failure of the first-line treatment (mTICl grade 0-2a).

$\S$ One missing value.

II Acute antiplatelet therapy was defined as the administration of at least 1 antiplatelet medication.

${ }^{* *}$ Permanent stenting is defined as placement of a stent in the setting of underlying atherosclerotic stenosis $>70 \%$ (NASCET).

MT in BAO. Similar reperfusion rates were observed in the Endovascular Stroke Treatment (ENDOSTROKE) study including 148 BAO patients (stent retriever treatment in $84 \%)^{27}$ and in the stent retriever thrombectomy meta-analysis including 312 subjects. ${ }^{11}$ A higher reperfusion rate was recently reported in a small series including 38 patients (89\%), 7 of whom had been treated only with intraarterial thrombolysis..$^{32}$ According to our results, successful reperfusion improved the clinical outcome and ADAPT led to a higher complete reperfusion rate, which appears to be a better predictor of a favorable outcome than mTICI grade $2 \mathrm{~b} .{ }^{6}$ To date, a direct comparison according to the type of first-line endovascular approach in $\mathrm{BAO}$ patients has been used in only 2 small, retrospective single-center studies including, respectively, 31 (18 aspirations vs 13 stent retrievers) $)^{28}$ and 33 (20 aspirations vs 13 stent retrievers) patients. ${ }^{9}$ In both studies, ADAPT seemed to allow more rapid and complete recanalization than stent retriever thrombectomy. A larger, retrospective multicenter series compared the results of stent retriever treatment and aspiration thrombectomy as primary approaches; unfortunately, however, only $48 \mathrm{BAO}$ patients were included in that study. ${ }^{19}$

The exact relationship between reperfusion and outcome is not fully resolved in BAO patients. In the ENDOSTROKE study, reperfusion per se did not predict a good outcome, ${ }^{27}$ whereas a strong effect of reperfusion on the rate of a good outcome was demonstrated in another series, ${ }^{32}$ as we found in our current study. To date, 2 randomized clinical trials have compared stent retrievers and ADAPT thrombectomy for large-vessel occlusions of the anterior circulation (Contact Aspiration vs Stent Retriever for Successful Revascularization [ASTER] study, NCT02523261; Comparison of Aspiration vs Stent Retriever as first-line approach [COMPASS], NCT02466893), and ADAPT did not result in better reperfusion in the ASTER trial. ${ }^{15}$ No data are available in the setting of posterior circulation strokes. Improving the technology of aspiration catheters 


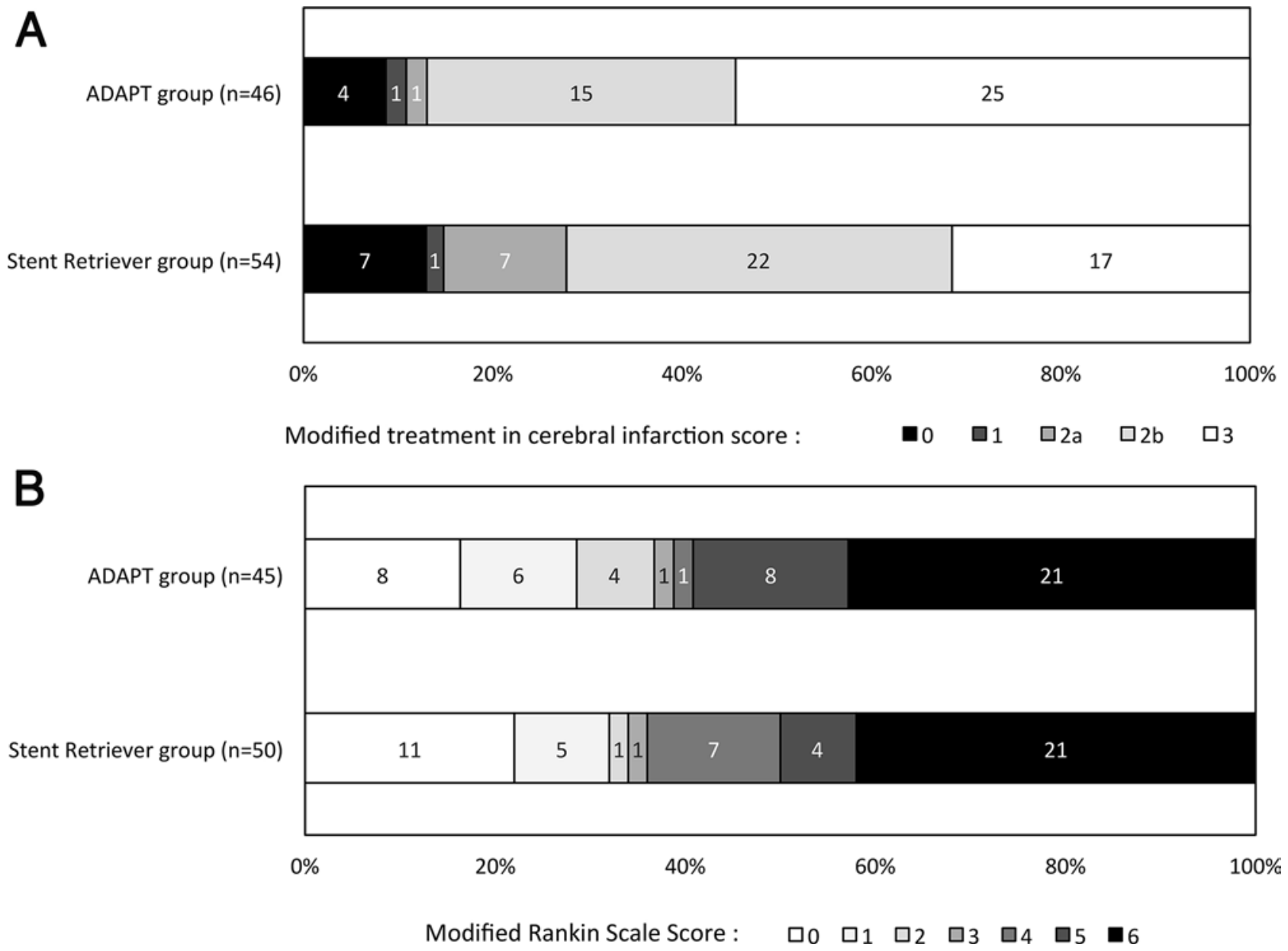

FIG. 3. Distribution of $\mathrm{mTICI}$ grade at the end of procedure $(\mathbf{A})$ and the $\mathrm{mRS}$ score at 90 days $(\mathbf{B})$, according to the first-line endovascular approach used.

may lead to better reperfusion and, subsequently, to better outcomes.

In our study, the rate of favorable outcomes was similar to that in previously published studies, ${ }^{11,19,27}$ although overall mortality was somewhat higher. However, a direct comparison is hampered by differences in individual clinical and imaging characteristics..$^{10}$ Indeed, patients undergoing MRI prior to reperfusion have significantly better clinical outcomes..$^{10,20,29}$ In our study, MRI-guided selection was performed in $73 \%$ of cases. Although a trend to-

TABLE 3. Angiographic and clinical efficacy outcomes

\begin{tabular}{|c|c|c|c|c|c|c|}
\hline \multirow[b]{2}{*}{ Parameter } & \multirow[b]{2}{*}{ Overall } & \multicolumn{2}{|c|}{ First-Line Endovascular Approach } & \multirow{2}{*}{$\begin{array}{c}\text { Effect } \\
\text { Size }\end{array}$} & \multirow[b]{2}{*}{ Value $(95 \% \mathrm{Cl})$} & \multirow{2}{*}{$\begin{array}{c}\mathrm{p} \\
\text { Value }\end{array}$} \\
\hline & & ADAPT & Stent Retriever & & & \\
\hline No. of patients & 100 & 46 & 54 & & & \\
\hline \multicolumn{7}{|l|}{ Reperfusion at end of procedure } \\
\hline $\mathrm{mTICl} 2 \mathrm{~b}-3^{*}$ & $79 / 100(79.0)$ & $40 / 46(87.0)$ & $39 / 54(72.2)$ & Odds ratio & $2.56(0.90$ to 7.29$)$ & 0.071 \\
\hline $\mathrm{mTICI} 3$ & $42 / 100(42.0)$ & $25 / 46(54.3)$ & $17 / 54(31.5)$ & Odds ratio & $2.59(1.14$ to 5.86$)$ & 0.021 \\
\hline \multicolumn{7}{|l|}{ Clinical outcome } \\
\hline Change in NIHSS score at $24 \mathrm{hrs} \dagger$ & $-3(-10$ to 4$)$ & $-3(-6$ to 1$)$ & $-2(-11$ to 7$)$ & SD $\ddagger$ & $0.08(-0.36$ to 0.55$)$ & 0.74 \\
\hline Favorable outcome: 90 -day mRS score 0-2 & $35 / 95(36.8)$ & $18 / 45(40.0)$ & $17 / 50(34.0)$ & Odds ratio & $1.29(0.56$ to 2.98$)$ & 0.55 \\
\hline 90-day all-cause mortality & $42 / 95(44.2)$ & $21 / 45(46.7)$ & $21 / 50(42.0)$ & Odds ratio & 1.21 (0.54 to 2.72$)$ & 0.65 \\
\hline
\end{tabular}

$\mathrm{SD}=$ standardized difference.

Values expressed as the number/total number (\%) or median (interquartile range), unless indicated otherwise.

* Prespecified as the primary efficacy outcome measure.

† Data available in 35 patients from the ADAPT group and 44 patients from the stent retriever group.

$\ddagger$ Calculated from nonparametric ANCOVA on rank-transformed data, adjusted for admission NIHSS score; $95 \%$ confidence interval was calculated using the bootstrap method (2000 bootstrap samples). 
TABLE 4. Comparison of angiographic and clinical efficacy outcomes between 2 endovascular approaches after handling missing values

\begin{tabular}{|c|c|c|c|c|c|c|c|}
\hline \multirow[b]{2}{*}{ Parameter } & \multicolumn{2}{|c|}{$\begin{array}{l}\text { First-Line Endovascular } \\
\text { Approach }\end{array}$} & \multirow[b]{2}{*}{$\begin{array}{l}\text { Effect } \\
\text { Size }\end{array}$} & \multirow{2}{*}{$\begin{array}{c}\text { Unadjusted } \\
\text { Value } \\
(95 \% \mathrm{Cl})\end{array}$} & \multirow[b]{2}{*}{$\begin{array}{c}p \\
\text { Value }\end{array}$} & \multirow{2}{*}{$\begin{array}{c}\text { Adjusted } \neq \\
\text { Value } \\
(95 \% \mathrm{Cl})\end{array}$} & \multirow[b]{2}{*}{$\begin{array}{c}p \\
\text { Value }\end{array}$} \\
\hline & ADAPT & $\begin{array}{c}\text { Stent } \\
\text { Retriever }\end{array}$ & & & & & \\
\hline No. of patients & 46 & 54 & & & & & \\
\hline \multicolumn{8}{|l|}{ Reperfusion at end of procedure } \\
\hline $\mathrm{mTICl} 2 \mathrm{~b}-3^{*}$ & $87.0 \%$ & $72.2 \%$ & Odds ratio & $2.56(0.90$ to 7.29$)$ & 0.071 & $2.34(0.80$ to 6.82$)$ & 0.12 \\
\hline \multicolumn{8}{|l|}{ Clinical outcome } \\
\hline Change in NIHSS score at $24 \mathrm{hrs}$ & $-1(-7$ to 14$)$ & $-3(-16$ to 7$)$ & $\mathrm{SD} \dagger$ & $0.22(-0.05$ to 0.73$)$ & 0.31 & $0.17(-0.12$ to 0.66$)$ & 0.42 \\
\hline 90-day favorable outcome: mRS score 0-2 & $41.3 \%$ & $36.3 \%$ & Odds ratio & $1.24(0.54$ to 2.80$)$ & 0.61 & 1.06 (0.36 to 3.02$)$ & 0.92 \\
\hline 90-day all-cause mortality & $45.7 \%$ & $38.9 \%$ & Odds ratio & $1.32(0.59$ to 2.93$)$ & 0.50 & $1.32(0.55$ to 3.13$)$ & 0.53 \\
\hline
\end{tabular}

$\mathrm{SD}=$ standardized difference.

Values expressed as percentage or median (interquartile range) calculated after handling missing data by multiple imputations, unless indicated otherwise.

* Prespecified as the primary efficacy outcome measure.

$\dagger$ Calculated from nonparametric ANCOVA on rank-transformed data, adjusted for admission NIHSS score; $95 \% \mathrm{Cl}$ was calculated using the bootstrap method (2000 bootstrap samples).

$\ddagger$ Adjusted for age, admission NIHSS score, diabetes, and time from symptom onset to groin puncture.

ward a better clinical outcome at 24 hours and 90 days was noted after ADAPT, the technique's clinical benefit was not demonstrated in our study, at least in part because of our small sample size, despite a higher successful reperfusion rate. A second explanation is that the relationship between clinical outcome and recanalization is weaker than that described in the anterior circulation, as reported in the ENDOSTROKE study. ${ }^{27}$ In the latter study, the authors demonstrated that the use of a stent retriever (the reference device in the present study) was associated with higher recanalization rates than non-stent-retriever devices.

Procedural complications occurred more frequently after stent retriever thrombectomy, especially in terms of new-territory embolic events. A low rate of complications due to embolization in a new territory has been previously described, ${ }^{4}$ although no difference was observed in the ASTER trial $(2.7 \%$ vs $3.7 \%) .{ }^{15}$ The systematic use of a balloon catheter probably explains the low rate of embolic complications after stent retriever thrombectomy in ASTER, as previously reported. ${ }^{21}$ However, the benefit of balloon catheter use for BAO thrombectomy is not yet established. Intracranial aspiration with proximal flow arrest by a balloon guiding catheter may also reduce the rate of embolic complications. ${ }^{18}$ Other safety parameters were similar between the aspiration and stent retriever groups in our study.

\section{Study Limitations}

The present study has several limitations. First, our findings are derived from observational nonrandomized analyses, which are subject to well-known limitations. We cannot rule out possible confounding effects by measured or unmeasured variables. Although our study included one of the largest reported series of stroke patients with BAO treated using MT, no formal study sample size was calculated. Thus, we cannot exclude that some differences may have been overlooked due to a lack of statistical power. In a posterior power calculation, we calculated the smallest significant between-group difference (expressed as effect size using the odds ratio) that our study sample size allowed us to detect with $80 \%$ power, assuming an incidence of outcome of $20 \%$ and $60 \%$ in the control group (stent retriever). Under these assumptions, only large differences could be detected with an OR of 3.38 and 3.66, respectively (or 0.30 and 0.27 for protective effects). Second, imaging outcomes were not evaluated by an independent committee across the 3 databases, even for the primary end point. Furthermore, the validity of mTICI grade has not been clearly approved for the posterior circulation, ${ }^{32}$ and mTICI has failed to achieve substantial interobserver agreement in posterior circulation stroke. ${ }^{14}$ In addition, various sizes of aspiration catheters and stent retrievers were used. For these reasons, the present findings can only be considered as hypothesis generating.

\section{Conclusions}

This study indicates that in BAO patients, successful reperfusion is a strong predictor of a 90-day favorable outcome and that ADAPT as the first-line strategy improves the rate of complete reperfusion and reduces procedure duration. These findings have potentially important implications for the current standards of practice in MT. Indeed, ADAPT may be a good option as a first-line approach in the setting of acute BAO since rapid and complete reperfusion is the major objective of acute ischemic stroke therapy. On the basis of these results, a randomized clinical trial directly comparing the $2 \mathrm{MT}$ strategies is warranted.

\section{Acknowledgments}

We thank Mary Osborne-Pellegrin for help in editing the final draft of the manuscript.

\section{Appendix \\ Endovascular Treatment In Ischemic Stroke (ETIS) \\ Research Investigators}

Jean-Pierre Decroix, Foch Hospital; Adrien Wang, Foch 
Hospital; Serge Evrard, Foch Hospital; Maya Tchikviladzé, Foch Hospital; Frederic Bourdain, Foch Hospital; Vadim Afanasiev, Foch Hospital; Loubna Majhadi, Foch Hospital; Bertrand Lapergue, Foch Hospital; Arthuro Consoli, Foch Hospital; Federico Di Maria, Foch Hospital; Oguzhan Coskun, Foch Hospital; Georges Rodesch, Foch Hospital; Delphine Lopez, Foch Hospital; Michel Piotin, Rothschild Foundation; Raphael Blanc, Rothschild Foundation; Hocine Redjem, Rothschild Foundation; Simon Escalard, Rothschild Foundation; Jean-Philippe Desilles, Rothschild Foundation; Gabriele Ciccio, Rothschild Foundation; Stanislas Smajda, Rothschild Foundation; Mikael Mazighi, Rothschild Foundation; Robert Fahed, Rothschild Foundation; Mickael Obadia, Rothschild Foundation; Candice Sabben, Rothschild Foundation; Guillaume Taylor, Rothschild Foundation; Malek Ben Maacha, Rothschild Foundation; Fernando Pico, Versailles Hospital; Haja Rakotoharinandrasana, Dreux Hospital; Philippe Tassan, Poissy Hospital; Roxanna Poll, Pontoise Hospital; Ovide Corabianu, Aulnay Hospital; Thomas de Broucker, Saint-Denis Hospital; Didier Smadja, Sud Francilien Hospital; Sonia Alamowitch, CHU Tenon; Mickael Obadia, Rothschild Foundation; Olivier Ille, Mantes la Jolie Hospital; Eric Manchon, Gonesse Hospital; Pierre-Yves Garcia, CHU Amiens; Roberto Riva, Hospices Civils de Lyon; Paul-Emile Labeyrie, Hospices Civils de Lyon; Norbert Nighoghossian, Hospices Civils de Lyon; Benjamin Gory, Hospices Civils de Lyon; Frédéric Philippeau, Bourg-en-Bresse Hospital; Serkan Cakmak, Nord Ouest Hospital; Karine Blanc-Lasserre, Valence Hospital; and Anne-Evelyne Vallet, Vienne Hospital.

\section{References}

1. Adams HP Jr, Bendixen BH, Kappelle LJ, Biller J, Love BB, Gordon DL, et al: Classification of subtype of acute ischemic stroke. Definitions for use in a multicenter clinical trial. TOAST. Trial of Org 10172 in Acute Stroke Treatment. Stroke 24:35-41, 1993

2. Bracard S, Ducrocq X, Mas JL, Soudant M, Oppenheim C, Moulin T, et al: Mechanical thrombectomy after intravenous alteplase versus alteplase alone after stroke (THRACE): a randomised controlled trial. Lancet Neurol 15:1138-1147, 2016

3. Buuren S, Groothuis-Oudshoorn K: mice: Multivariate imputation by chained equations in R. J Stat Softw 45:i03, 2011

4. Chueh JY, Puri AS, Wakhloo AK, Gounis MJ: Risk of distal embolization with stent retriever thrombectomy and ADAPT. J Neurointerv Surg 8:197-202, 2016

5. Conover WJ, Iman RL: Analysis of covariance using the rank transformation. Biometrics 38:715-724, 1982

6. Dargazanli C, Consoli A, Barral M, Labreuche J, Redjem H, Ciccio G, et al: Impact of modified TICI 3 versus modified TICI $2 b$ reperfusion score to predict good outcome following endovascular therapy. AJNR Am J Neuroradiol 38:90-96, 2017

7. Dennis M, Mead G, Doubal F, Graham C: Determining the modified Rankin score after stroke by postal and telephone questionnaires. Stroke 43:851-853, 2012

8. Efron B, Tibshirani RJ: An Introduction to the Bootstrap. New York: Chapman \& Hall, 1993

9. Gerber JC, Daubner D, Kaiser D, Engellandt K, Haedrich K, Mueller A, et al: Efficacy and safety of direct aspiration first pass technique versus stent-retriever thrombectomy in acute basilar artery occlusion-a retrospective single center experience. Neuroradiology 59:297-304, 2017

10. Gilberti N, Gamba M, Premi E, Costa A, Vergani V, Delrio I, et al: Endovascular mechanical thrombectomy in basilar artery occlusion: variables affecting recanalization and outcome. J Neurol 263:707-713, 2016

11. Gory B, Eldesouky I, Sivan-Hoffmann R, Rabilloud M, Ong E, Riva R, et al: Outcomes of stent retriever thrombectomy in basilar artery occlusion: an observational study and system- atic review. J Neurol Neurosurg Psychiatry 87:520-525, 2016

12. Goyal M, Menon BK, van Zwam WH, Dippel DW, Mitchell PJ, Demchuk AM, et al: Endovascular thrombectomy after large-vessel ischaemic stroke: a meta-analysis of individual patient data from five randomised trials. Lancet 387:17231731,2016

13. Hacke W, Kaste M, Fieschi C, von Kummer R, Davalos A, Meier D, et al: Randomised double-blind placebo-controlled trial of thrombolytic therapy with intravenous alteplase in acute ischaemic stroke (ECASS II). Lancet 352:1245-1251, 1998

14. Jung C, Yoon W, Ahn SJ, Choi BS, Kim JH, Suh SH: The revascularization scales dilemma: is it right to apply the treatment in cerebral ischemia scale in posterior circulation stroke? AJNR Am J Neuroradiol 37:285-289, 2016

15. Lapergue B, Blanc R, Gory B, Labreuche J, Duhamel A, Marnat G, et al: Effect of endovascular contact aspiration vs stent retriever on revascularization in patients with acute ischemic stroke and large vessel occlusion: the ASTER randomized clinical trial. JAMA 318:443-452, 2017

16. Lapergue B, Blanc R, Guedin P, Decroix JP, Labreuche $\mathrm{J}$, Preda C, et al: A direct aspiration, first pass technique (ADAPT) versus stent retrievers for acute stroke therapy: an observational comparative study. AJNR Am J Neuroradiol [epub ahead of print], 2016

17. Liu X, Xu G, Liu Y, Zhu W, Ma M, Xiong Y, et al: Acute basilar artery occlusion: Endovascular Interventions versus Standard Medical Treatment (BEST) Trial-Design and protocol for a randomized, controlled, multicenter study. Int J Stroke 12:779-785, 2017

18. Matsumoto H, Nishiyama H, Tetsuo Y, Takemoto H, Nakao $\mathrm{N}$ : Initial clinical experience using the two-stage aspiration technique (TSAT) with proximal flow arrest by a balloon guiding catheter for acute ischemic stroke of the anterior circulation. J Neurointerv Surg [epub ahead of print], 2016

19. Mokin M, Sonig A, Sivakanthan S, Ren Z, Elijovich L, Arthur A, et al: Clinical and procedural predictors of outcomes from the endovascular treatment of posterior circulation strokes. Stroke 47:782-788, 2016

20. Nagel S, Herweh C, Köhrmann M, Huttner HB, Poli S, Hartmann M, et al: MRI in patients with acute basilar artery occlusion - DWI lesion scoring is an independent predictor of outcome. Int J Stroke 7:282-288, 2012

21. Nguyen TN, Malisch T, Castonguay AC, Gupta R, Sun CH, Martin CO, et al: Balloon guide catheter improves revascularization and clinical outcomes with the Solitaire device: analysis of the North American Solitaire Acute Stroke Registry. Stroke 45:141-145, 2014

22. Nogueira RG, Lutsep HL, Gupta R, Jovin TG, Albers GW, Walker GA, et al: Trevo versus Merci retrievers for thrombectomy revascularisation of large vessel occlusions in acute ischaemic stroke (TREVO 2): a randomised trial. Lancet 380:1231-1240, 2012

23. Rubin DB: Multiple Imputation for Nonresponse in Surveys. New York: Wiley, 1987

24. Saver JL, Jahan R, Levy EI, Jovin TG, Baxter B, Nogueira $\mathrm{RG}$, et al: Solitaire flow restoration device versus the Merci Retriever in patients with acute ischaemic stroke (SWIFT): a randomised, parallel-group, non-inferiority trial. Lancet 380:1241-1249, 2012

25. Schonewille WJ, Wijman CAC, Michel P, Rueckert CM, Weimar C, Mattle HP, et al: Treatment and outcomes of acute basilar artery occlusion in the Basilar Artery International Cooperation Study (BASICS): a prospective registry study. Lancet Neurol 8:724-730, 2009

26. Schulz UG, Fischer U: Posterior circulation cerebrovascular syndromes: diagnosis and management. J Neurol Neurosurg Psychiatry 88:45-53, 2017 
27. Singer OC, Berkefeld J, Nolte CH, Bohner G, Haring HP, Trenkler J, et al: Mechanical recanalization in basilar artery occlusion: the ENDOSTROKE study. Ann Neurol 77:415424, 2015

28. Son S, Choi DS, Oh MK, Hong J, Kim SK, Kang H, et al: Comparison of Solitaire thrombectomy and Penumbra suction thrombectomy in patients with acute ischemic stroke caused by basilar artery occlusion. J Neurointerv Surg 8:13-18, 2016

29. Strbian D, Sairanen T, Silvennoinen H, Salonen O, Kaste M, Lindsberg PJ: Thrombolysis of basilar artery occlusion: impact of baseline ischemia and time. Ann Neurol 73:688-694, 2013

30. Turk AS, Spiotta A, Frei D, Mocco J, Baxter B, Fiorella D, et al: Initial clinical experience with the ADAPT technique: a direct aspiration first pass technique for stroke thrombectomy. J Neurointerv Surg 6:231-237, 2014

31. van der Hoeven EJ, Schonewille WJ, Vos JA, Algra A, Audebert HJ, Berge E, et al: The Basilar Artery International Cooperation Study (BASICS): study protocol for a randomised controlled trial. Trials 14:200, 2013

32. van Houwelingen RC, Luijckx GJ, Mazuri A, Bokkers RP, Eshghi OS, Uyttenboogaart M: Safety and outcome of intraarterial treatment for basilar artery occlusion. JAMA Neurol 73:1225-1230, 2016
33. Zaidat OO, Yoo AJ, Khatri P, Tomsick TA, von Kummer R, Saver JL, et al: Recommendations on angiographic revascularization grading standards for acute ischemic stroke: a consensus statement. Stroke 44:2650-2663, 2013

\section{Disclosures}

Dr. Mazighi has been a consultant for Medtronic. Dr. Turjman has been a consultant for Codman, Balt, and Medtronic.

\section{Author Contributions}

Conception and design: Gory, Lapergue. Acquisition of data: Gory, Mazighi, Blanc, Piotin, Turjman, Lapergue. Analysis and interpretation of data: Gory, Mazighi, Blanc, Piotin, Turjman, Lapergue. Drafting the article: Gory. Critically revising the article: Gory, Mazighi, Lapergue. Reviewed submitted version of manuscript: Gory, Mazighi, Blanc, Piotin, Turjman, Lapergue. Approved the final version of the manuscript on behalf of all authors: Gory. Statistical analysis: Labreuche. Study supervision: Gory, Lapergue.

\section{Correspondence}

Benjamin Gory: CHRU Nancy, Hôpital Central, Nancy, France. b.gory@chru-nancy.fr. 